\title{
Surgical Adrenalectomy with Diurnal Corticosterone Replacement Slows Escalation and Prevents the Augmentation of Cocaine-Induced Reinstatement in Rats Self-Administering Cocaine Under Long-Access Conditions
}

\author{
John R Mantsch*', David A Baker', Joseph P Serge', Michael A Hoks', David M Francis' and Eric S Katz' \\ 'Department of Biomedical Sciences, Marquette University, Milwaukee, WI, USA
}

\begin{abstract}
The loss of control over cocaine use and persistently heightened susceptibility to drug relapse that define human cocaine addiction are consequences of drug-induced neuroplasticity and can be studied in rats self-administering cocaine under conditions of daily long access ( $\operatorname{Lg} A)$ as escalating patterns of drug intake and heightened susceptibility to reinstatement. This study investigated the potential contribution of elevated glucocorticoids at the time of LgA cocaine self-administration (SA) to these behavioral indices of addictionrelated neuroplasticity. Rats provided 14 days of 6-h access ( $\mathrm{LgA})$ to cocaine showed a progressive escalation of SA and were more susceptible to cocaine-induced reinstatement ( $10 \mathrm{mg} / \mathrm{kg}$, i.p.) compared to rats self-administering under short-access (ShA; $2 \mathrm{~h}$ ) conditions. A surgical adrenalectomy and corticosterone replacement (ADX/C) regimen that eliminated SA-induced increases in corticosterone (CORT) while maintaining the diurnal pattern of secretion failed to alter SA or reinstatement in ShA rats but slowed escalation and attenuated later reinstatement in LgA rats when applied before but not after chronic LgA SA testing. Although the contribution of other adrenal hormones cannot be ruled out, these data suggest that elevated glucocorticoids at the time of cocaine exposure may be required for the effects of LgA SA on cocaine intake and later reinstatement. The inability of daily CORT administration before daily ShA SA, at a dose that reproduced the response during LgA SA, to mimic the effects of LgA SA suggests that elevated glucocorticoids during SA may play a permissive role in cocaine-induced neuroplasticity that contributes to addiction. Neuropsychopharmacology (2008) 33, 8I4-826; doi:I0.1038/sj.npp. I30 I464; published online 30 May 2007
\end{abstract}

Keywords: addiction; neuroplasticity; corticosterone; escalation; relapse; cocaine

\section{INTRODUCTION}

The unpredictable relapse of cocaine use that occurs even after extended periods of drug abstinence is the primary obstacle to the effective management of cocaine addiction. This chronically relapsing nature of cocaine addiction is likely attributable to long-lasting, if not permanent, neuroplasticity that emerges as a consequence of repeated drug use. Understanding the neuroplasticity that determines susceptibility to drug relapse is essential for the development of new and more effective therapeutic interventions aimed at minimizing the risk that renewed drug use will occur following detoxification.

One approach that has been used to investigate cocaineinduced neuroplasticity that is pathogenic for addiction has

*Correspondence: Dr JR Mantsch, Department of Biomedical Sciences, Marquette University, Schroeder Health Complex, PO Box I88I, Milwaukee, WI 5320I, USA, Tel: + | 4 |4 288 2036, Fax: + | 414288 6564, E-mail: john.mantsch@marquette.edu

Received 25 September 2006; revised 26 April 2007; accepted 27 April 2007 involved the study of rats provided chronic daily prolonged access to cocaine for self-administration (SA). Rats provided long access to cocaine for SA each day (longaccess; LgA rats), but not rats provided shorter drug access (short-access; ShA rats), display a progressive escalation of cocaine intake suggested to be related to the loss of control over drug use that is central to human cocaine addiction (Ahmed and Koob, 1998, 1999). We and others have demonstrated that $\operatorname{LgA}$ rats also display greater reinstatement following administration of cocaine (Mantsch et al, 2004; Ahmed and Cador, 2006), cocaine-associated cues (Kippin et al, 2006), and a stressor (electric footshock; unpublished observation) when measured several weeks after SA testing compared to ShA rats. Thus, cocaine SA appears to produce long-term neuroadaptations that lead to a heightened susceptibility to engage in cocaine-seeking behavior and emerge in an intake-dependent manner.

Glucocorticoids secreted as a consequence of activation of the stressor-responsive hypothalamic-pituitary-adrenal (HPA) axis play a critical role in physiological processes that enable organisms to effectively adapt to and cope with 
stressors (de Kloet et al, 2005; McEwen, 2005). Although elevated glucocorticoids are of short-term benefit to an organism, prolonged and/or repeated elevations of glucocorticoids during periods of chronic stress are maladaptive and lead to a number of pathological conditions, some of which may contribute to the addiction process. For example, it has been reported that elevated corticosterone (CORT) during periods of chronic or repeated stress is involved in the stressor-induced facilitation of cocaine SA (Goeders and Guerin, 1996a,b; Mantsch et al, 1998; Campbell and Carroll, 2001) and in the stressor-induced sensitization of the locomotor stimulating effects of cocaine (Prasad et al, 1998; Rouge-Pont et al, 1995). More recently, we have demonstrated that daily exposure to a stressor, uncontrollable electric footshock stress, at the time of SA testing produces a progressive escalation of cocaine SA that is dependent on elevated glucocorticoids, suggesting that the neuroplasticity that contributes to heightened drugseeking behavior in cocaine addicts may be glucocorticoid dependent (Mantsch and Katz, 2007).

Like stressors, self-administered cocaine increases glucocorticoid secretion in rats (Galici et al, 2000), nonhuman primates (Broadbear et al, 1999), and human cocaine addicts (Ward et al, 1999; Ghitza et al, 2007). When rats self-administer cocaine during LgA sessions, increases in plasma CORT are greater and more persistent than they are in ShA rats (Mantsch et al, 2003) and physiological signs of increased glucocorticoid secretion (eg reductions in thymus weight and adrenal hypertrophy) are exaggerated (unpublished observation). Thus, any contribution of elevated glucocorticoids to cocaine-induced neuroplasticity should be particularly pronounced in rats self-administering cocaine under LgA conditions.

The goal of the present study was to investigate how elevation of glucocorticoids as a consequence of cocaine SA under $\operatorname{LgA}$ conditions contributes to addiction-related neuroplasticity expressed as escalating patterns of cocaine intake and augmented cocaine-induced reinstatement. The role of elevated glucocorticoids was examined by surgically adrenalectomizing rats and then providing them with hormone replacement using a protocol that maintains diurnal patterns of plasma CORT while eliminating evoked increases in CORT secretion (Mantsch and Katz, 2007). We hypothesized that by eliminating the elevation of glucocorticoids during cocaine SA, we would not alter cocaine SA and cocaine-induced reinstatement in ShA rats but would impair the escalation of SA under LgA conditions and the resulting augmentation of cocaine-induced reinstatement.

\section{METHODS}

\section{Subjects}

One-hundred and nine adult male Sprague-Dawley rats (Harlan Laboratories Inc., St Louis, MO), approximately 90 days old ( $325 \mathrm{~g}$ ) were used for the study. Rats were housed individually in a temperature- and humidity-controlled, AAALAC-accredited animal facility under a $12 / 12 \mathrm{~h}$ reversed light/dark cycle (lights on at 1800) and had access to food ad libitum, except when in the experimental chambers. Water was also available ad libitum, including in the experimental chambers. All procedures were carried out in accordance with the Guide for the Care and Use of Laboratory Animals as adopted and promulgated by the National Institutes of Health.

\section{Catheterization Surgery}

Rats were implanted with chronic indwelling catheters under ketamine $\mathrm{HCl}(100 \mathrm{mg} / \mathrm{kg}$, i.p.; Fort Dodge Animal Health, Fort Dodge, IA) and xylazine ( $2 \mathrm{mg} / \mathrm{kg}$, i.p.; Lloyd Laboratories, Shenandoah, IA) anesthesia. A silicon-tubing catheter (Silastic, Dow Corning Co., Midland, MI; $0.64 \mathrm{~mm}$ i.d.; $1.19 \mathrm{~mm}$ o.d.) was inserted into the right posterior facial vein and down into the jugular vein so that it terminated at the right atrium. The catheter was sutured to the vein and continued subcutaneously to the animal's back where it exited $2 \mathrm{~cm}$ posterior to the scapula via a back-mounted 22-gauge guide cannula (Plastics One Inc., Roanoke, VA) attached using dental acrylic to a piece of polypropylene monofilament surgical mesh (Atrium Medical, Co., Hudson, $\mathrm{NH}$ ) to permit connection of a polyethylene delivery line ( $0.58 \mathrm{~mm}$ i.d. $\times 1.27 \mathrm{~mm}$ o.d.; Plastics One Inc.) encased in a stainless steel spring leash (Plastics One Inc.). The delivery line was connected to a $30-\mathrm{ml}$ syringe in a motor-driven pump (Razel, Stamford, CT) via a leakproof fluid swivel (Instech Lab. Inc., Plymouth Meeting, PA) suspended above the chamber to allow drug solution delivery. The swivel and leash assembly was counterbalanced to permit relatively unrestrained movement. Rats were allowed to recover for at least 3 days before SA testing during which time they were provided acetaminophen $(480 \mathrm{mg} / \mathrm{l})$ in their drinking water. After implantation, rats were injected with a sterile cefazolin antibiotic solution (15 mg, i.v.; West-Ward Pharmaceutical Co., Eatontown, NJ) each day. Catheters were filled daily with a heparin solution (83 i.u./ml; Elkins-Sinn Inc., Cherry Hill, NJ) and capped whenever the leash/delivery line assembly was disconnected.

\section{SA Apparatus}

Twenty plastic and stainless steel operant conditioning chambers encased in sound attenuating cubicles (MEDAssociates Inc., St Albans, VT) were used. Chambers were equipped with retractable levers with stimulus lights located above them and water bottles. One lever and its stimulus light were mounted on the front wall of the chamber. A second lever and light were located on the back wall. Cubicles were equipped with exhaust fans that provided ventilation and white noise to mask extraneous sound.

\section{SA Training/Testing}

Following recovery from surgery, rats were trained to selfadminister cocaine $(1.0 \mathrm{mg} / \mathrm{kg} / \mathrm{inf}$, i.v., National Institute on Drug Abuse, Bethesda, MD) by pressing a lever under a fixed radio 1 (FR1) schedule during daily 2 -h sessions, within which the active (ie front) lever was extended into the chamber and the corresponding stimulus light was illuminated. Depression of this lever resulted in an i.v. infusion of drug solution $(200 \mu$ delivered over $5 \mathrm{~s})$ followed by a 25 -s time-out period during which the stimulus light was extinguished but the lever remained extended. Responding on a second, inactive (ie back) lever was recorded 
but had no programmed consequences. Once significant SA under the FR1 schedule was observed (ie $>10$ infusions), the response requirements for SA were increased to FR2, and then to FR4 using the same criterion. Once a stable response pattern was observed under the FR4 schedule (total responding $<10 \%$ variation from the mean over three consecutive sessions), rats were ready for SA testing.

\section{ADX and Diurnal CORT Replacement}

To investigate the involvement of elevated CORT in cocaine $\mathrm{SA}$, extinction, and reinstatement, some rats (ADX/C rats) were bilaterally adrenalectomized via the dorsal approach under ketamine and xylazine anesthesia and received diurnal CORT replacement. Diurnal CORT replacement consisted of subcutaneous implantation of a $25 \%$ CORT (Sigma Chemicals, St Louis, MO) pellet in the nape of the neck to produce blood concentrations similar to those found at the nadir of the diurnal cycle along with inclusion of $0.025 \%$ CORT in the drinking water to emulate the circadian peak observed during the active (dark) phase, within which most drinking occurs (Jacobson et al, 1988). Twenty-five percent CORT pellets were made by melting a 1:3 CORT:cholesterol ratio mixture over a flame and pouring it into a pellet mold, creating a $1.5 \times 0.5 \times 0.5 \mathrm{~cm}$ $(\mathrm{l} \times \mathrm{w} \times \mathrm{h})$ pellet for implantation (Meyer et al, 1979). CORT was dissolved in ethanol before introduction into the drinking water, resulting in a final ethanol concentration of $0.0001 \%$. To replace depleted sodium secondary to the loss of aldosterone as a result of ADX, drinking water for all rats consisted of a $0.9 \% \mathrm{NaCl}$ solution. CORT pellets were replaced every 7 days under brief anesthesia induced by sodium methohexital (1.5 mg, i.v., Monarch Pharmaceuticals, Bristol, TN) injected via implanted catheter. Control (sham) rats underwent sham ADX procedures and were implanted with $100 \%$ cholesterol pellets. Sham operations were identical to ADX surgeries except that, once exposed, the adrenal glands were not removed during the procedure.

\section{Experimental Time Line for Experiments 1-2}

The time line for experiments 1-3 is shown in Table 1.

\section{Experiment 1: Effects of Extended Drug Access on Cocaine SA, Extinction, and Cocaine-Induced Reinstatement}

The effects of LgA SA on cocaine intake, extinction, and later cocaine-induced reinstatement were examined in 40 rats. Once stable SA was observed in these rats, they underwent $\mathrm{ADX} / \mathrm{C}$ sham treatment as described above. Upon recovery from surgery, baseline SA was determined before assignment of rats to one of two treatment groups according to the conditions under which cocaine was available for SA for the next 14 days. ShA rats $(n=20)$ continued to have access to cocaine for SA during daily 2-h sessions. LgA rats $(n=20)$ were provided access to cocaine during daily 6-h sessions across the 14-day SA testing period. After 14 days of ShA or LgA SA, lever-pressing was extinguished by replacing the cocaine solution with saline for 10 consecutive 2 -h extinction sessions. These sessions were otherwise identical to the ShA SA sessions. Before the

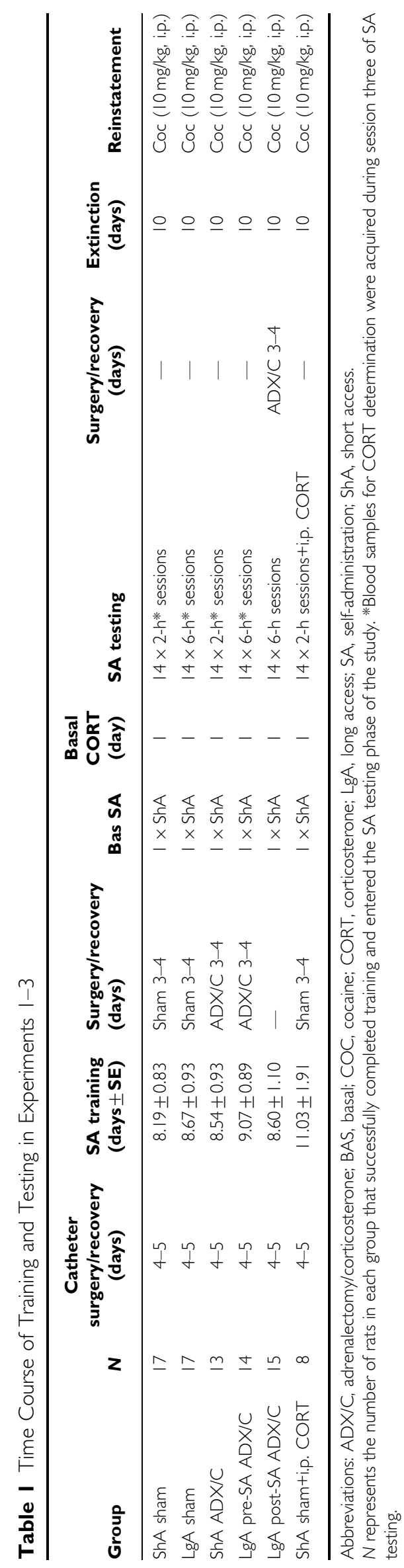


final (day 10) extinction session, rats were injected with saline $(1 \mathrm{mg} / \mathrm{ml}, 0.9 \% \mathrm{NaCl}$, i.p.). Cocaine-induced reinstatement was examined during the next 2 -h session, before which rats received an injection of cocaine $(10 \mathrm{mg} / \mathrm{kg}$ in saline, $1 \mathrm{mg} / \mathrm{ml}$, i.p.).

\section{Experiment 2: Effects of Surgical ADX with Diurnal CORT Replacement (ADX/C) on SA, Extinction, and Reinstatement in ShA and LgA Rats}

The role of cocaine-induced increases in CORT in cocaine $\mathrm{SA}$, extinction, and reinstatement was investigated using 54 $\mathrm{ADX} / \mathrm{C}$ rats. Once stable SA was observed, 36 of these rats underwent $\mathrm{ADX} / \mathrm{C}$ and were assigned to $\mathrm{ShA}$ and $\operatorname{LgA}$ groups (pre-SA ShA ADX/C, $n=18$; pre-SA LgA ADX/C, $n=18$ ). After 14 days of ShA or LgA SA, these rats went through extinction and were tested for reinstatement. SA, extinction, and reinstatement in these rats were compared with SA, extinction, and reinstatement in the sham-treated ShA (pre-SA ShA sham) and LgA (pre-SA LgA sham) rats described in Experiment 1. To determine if eliminating CORT responsiveness after the completion of SA testing through ADX/C altered extinction and reinstatement in a manner similar to ADX/C before LgA SA testing, a fifth group of rats $(n=18)$ was tested. These rats underwent LgA SA as described above with no prior surgery and then received $\mathrm{ADX} / \mathrm{C}$ after 14 days of LgA SA testing and before extinction. These rats were allowed to recover for 3-4 days before the 10-day extinction period. For these rats, extinction, and reinstatement were otherwise identical to the other treatment groups.

\section{Experiment 3: Effects of Daily i.p. CORT Injections at the Time of ShA SA on Cocaine SA, Extinction, and Reinstatement}

To determine if we could mimic the effects of LgA SA by producing comparable increases in CORT in ShA rats, we tested the effects of i.p. CORT administration before daily ShA SA testing on cocaine SA and reinstatement. Ten ShA rats received daily i.p. injections with CORT $(2.0 \mathrm{mg} / \mathrm{kg})$ suspended in extra virgin olive oil for 14 days immediately before daily SA. We have previously demonstrated that this CORT dosing regimen facilitates the acquisition of cocaine SA in rats (Mantsch et al, 1998). Preliminary studies indicated that the pattern of elevated CORT following administration of this CORT dose resembles the persistent elevations of CORT that are observed in rats self-administering under LgA but not ShA conditions (Mantsch et al, 2003). After SA training, these rats underwent ShA SA testing with i.p. CORT pretreatment for 14 days and then went through extinction training and were tested for cocaine-induced reinstatement in the absence of CORT injections. It was hypothesized that if elevated CORT at the time of SA testing was sufficient for the neuroplasticity leading to escalated cocaine SA and augmented reinstatement observed in LgA rats, ShA rats tested for SA following CORT pretreatment should escalate their SA and show increased reinstatement compared to ShA rats tested in the absence of the CORT injections.

\section{Plasma CORT Determination}

Blood samples (approximately $500 \mu \mathrm{l}$ ) were acquired from the catheters of some $\mathrm{ADX} / \mathrm{C}$ and sham-treated rats under basal conditions the day before SA testing was initiated and at various times before and following the ShA and LgA SA sessions on day 3 of SA testing for determination of plasma CORT concentrations using a commercial radioimmunoassay kit (MP Biochemicals, Irvine, CA). An additional five rats were trained to self-administer cocaine so that the plasma CORT levels in rats receiving an i.p. CORT injection $(2.0 \mathrm{mg} / \mathrm{kg})$ before a ShA SA could be determined. These rats were separate from the rats used for determination of the effects of repeated CORT administration on SA and reinstatement. Blood was collected in tubes containing heparin, stored on ice, and centrifuged to allow separation of plasma, which was frozen at $-80^{\circ} \mathrm{C}$. Plasma samples were assayed in duplicate ( $10 \mu \mathrm{l}$ per duplicate) in three batches. The mean coefficient of intra-assay variation determined from replicates of randomly chosen samples analyzed in the same assay was $7.65 \%(n=3)$. The mean coefficient of interassay variation determined from replicates of randomly chosen samples analyzed in different assays (ie batches) was $15.19 \%(n=3)$.

\section{Statistical Analyses}

Statistical analyses were performed using SPSS 14.0 software (Chicago, IL). The statistical significance of differences within and among groups was determined using analysis of variance (ANOVA) followed, when appropriate, with further analyses of simple main effects using ANOVA and/or post hoc testing using the Fisher's least significant difference test. For all analyses, statistical significance was defined as $P<0.05$.

\section{RESULTS}

\section{Experiment 1: Effects of Extended Drug Access on Cocaine SA, Extinction, and Cocaine-Induced Reinstatement}

Cocaine SA, extinction, and cocaine-induced reinstatement in $\mathrm{ADX} / \mathrm{C}$ sham-treated rats are shown in Figure 1. Seventeen of the 20 sham-treated ShA rats completed the study. Two ShA rats were excluded due to catheter failure, and one excluded due to illness. Seventeen of the 20 shamtreated LgA rats completed the SA component of the study. Two LgA rats were excluded due to catheter failure and one died of unknown causes. Additionally, two LgA rats died following illness during extinction testing, resulting in $n=15$ for the extinction and reinstatement components of the experiment.

Self-administration. No differences in the rate of acquisition of SA ( $\mathrm{ShA}=8.19 \pm 0.83$ days $v s \mathrm{LgA}=8.67 \pm 0.93$ days $)$ or $\mathrm{SA}$ on the final day of training ( $\mathrm{ShA}=15.32 \pm 1.15$ infusions $v s \operatorname{LgA}=14.30 \pm 0.92$ infusions) were observed between $\mathrm{ADX} / \mathrm{C}$ sham-treated ShA and LgA rats (two-tailed $t$-tests).

Figure 1a shows cocaine $\mathrm{SA}$ in $\mathrm{ADX} / \mathrm{C}$ sham-treated ShA and LgA rats. As previously demonstrated, rats self- 

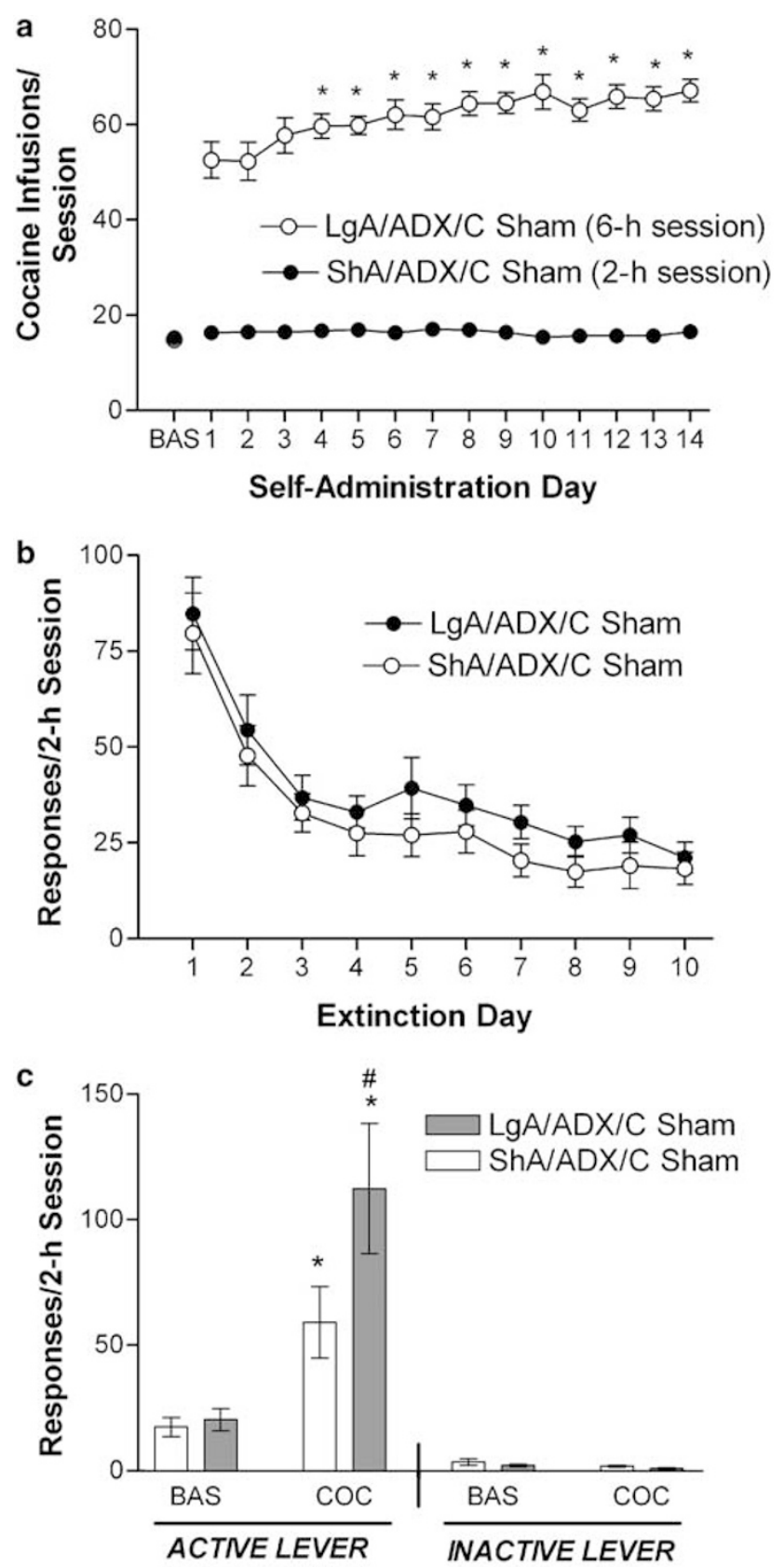

Figure I Cocaine SA (a), extinction (b), and cocaine-induced reinstatement (c) in ADX/C sham-treated ShA and LgA rats. Data in (a) represent cocaine SA (infusions per session \pm SE) under basal conditions (BAS) and across the 14 days of SA testing in ShA and LgA rats. Significant escalation of cocaine SA was observed in LgA, but not ShA, rats (*significant $P<0.05$ vs SA day I). Data in (b) represent daily responding across the 10-day extinction period. Significant differences in extinction were not observed between ShA and LgA rats (b). (c) Responding by ShA and LgA rats on the previously active and inactive levers following cocaine administration $(10 \mathrm{mg} / \mathrm{kg}$, i.p.) during reinstatement testing is shown. Cocaine increased responding on the previously active but not inactive lever in both ShA and $L g A$ rats (*significant $P<0.05$ vs SAL/BAS). Cocaine-induced reinstatement was significantly greater in LgA rats compared to ShA rats (" ${ }^{\#}$ significant $P<0.05)$.

administering cocaine under $\operatorname{LgA}$, but not ShA conditions displayed a progressive escalation of cocaine intake. A two-way access condition (ShA $v s \mathrm{LgA}) \times \mathrm{SA}$ day (repeated

measure; day 1-14) ANOVA revealed significant overall effects of access condition $\left(\mathrm{F}_{13,403}=6.23 ; P<0.001\right)$ and SA day $\left(\mathrm{F}_{13,403}=7.34 ; P<0.001\right)$ and a significant access condition $\times$ SA day interaction $\left(\mathrm{F}_{1,31}=339.84 ; P<0.001\right)$. Post hoc examination of simple main effects using one-way ANOVA of SA in ShA and LgA rats showed a significant escalation of cocaine $\mathrm{SA}$ in $\operatorname{LgA}$ rats $\left(\mathrm{F}_{13,195}=6.89\right.$; $P<0.001$ ), but not ShA rats. Cocaine SA was significantly increased on SA days 4-14 compared to SA day 1 in $\operatorname{LgA}$ rats $(P<0.05)$.

Extinction. Responding during extinction in ADX/C shamtreated ShA and LgA rats is shown in Figure 1b. A two-way ANOVA showed a significant overall effect of extinction day but failed to show a significant effect of SA group or a significant interaction between SA group and extinction day. Lever-pressing progressively decreased across extinction testing in both ShA and LgA sham-treated rats, but did not significantly differ between the two groups.

Reinstatement. Cocaine-induced reinstatement in shamtreated ShA and LgA rats is shown in Figure 1c. A two-way access $($ ShA $v s \quad \operatorname{LgA}) \times$ reinstatement condition (saline $v s$ cocaine; repeated measure) ANOVA showed significant overall effects of reinstatement $\left(\mathrm{F}_{1,28}=32.35 ; P<0.001\right)$ and access condition $\left(\mathrm{F}_{1,28}=4.80 ; P<0.05\right)$, and a significant reinstatement $\times$ access condition interaction $\left(\mathrm{F}_{1,28}=5.44\right.$; $P<0.05)$. Significant cocaine-induced reinstatement was observed in both ShA and $\operatorname{LgA}$ rats $(P<0.01$ for each group). However, the magnitude of reinstatement was significantly greater in $\operatorname{LgA}$ rats compared to ShA rats. Analysis of simple main effects using two-tailed $t$-tests showed that cocaine-induced reinstatement, but not responding following a saline injection on extinction day 10 was significantly greater in $\operatorname{LgA}$ compared to ShA rats $\left(t_{28}=27.34 ; P<0.05\right)$. Inactive lever-pressing by ShA and LgA rats is also shown in Figure 1c. ANOVA failed to show significant effects of, or interaction between, the reinstatement and SA conditions for inactive lever responding.

\section{Experiment 2: Effects of Surgical ADX with Diurnal CORT Replacement (ADX/C) on SA, Extinction, and Reinstatement in ShA and LgA Rats}

The role of elevated glucocorticoids in cocaine SA, extinction, and cocaine-induced reinstatement in ShA and LgA rats was tested using $\mathrm{ADX} / \mathrm{C}$ rats.

Plasma CORT. Plasma CORT levels under basal conditions and during ShA and LgA SA in ADX/C and sham-treated rats are shown in Table 2. The effects of SA condition (ShA SA, LgA SA, baseline), ADX/C condition (ADX/C, sham), and time of sampling $(0700,0930$, and 1400) were examined using three-factor univariate ANOVAs. Since CORT samples were not acquired from LgA rats at the 0930 time-point, two separate ANOVAs were used to compare CORT levels: one examining CORT levels at 0700 and 0930 only in ADX/C and sham-treated ShA rats; and one examining CORT levels at 0700 and 1400 in ADX/C and sham-treated ShA and LgA rats. In each case, a significant interaction between ADX/C condition, SA condition, and time of testing was observed $\left(\mathrm{F}_{1,86}=3.94 ; P=0.05\right.$ for the ANOVA including the 0930 
Table 2 Plasma CORT (ng/ml \pm SE) Under Basal Conditions and during ShA and LgA SA Testing in ADX/C and Sham-Treated Rats

\begin{tabular}{|c|c|c|c|c|c|c|}
\hline & \multicolumn{3}{|c|}{ Sham } & \multicolumn{3}{|c|}{$A D X / C$} \\
\hline & 0700 & 0930 & 1400 & 0700 & 0930 & 1400 \\
\hline ShA & $136.85 \pm 18.65$ & $236.80 * \pm 12.58$ & $80.22 \pm 12.73$ & $156.68 \pm 21.67$ & $1 \mid 6.24 \pm 41.23$ & $94.38 \pm 7.75$ \\
\hline LgA & $124.73 \pm 19.73$ & - & |84.89*** \pm 5.54 & $|47.94 \pm 24.3|$ & - & $90.06 \pm 11.77$ \\
\hline
\end{tabular}

Abbreviations: ADX/C, adrenalectomy/corticosterone; BAS, basal; CORT, corticosterone; LgA, long access; SA, self-administration; ShA, short access.

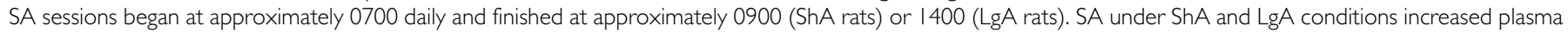
CORT in sham- but not ADX/C-treated rats. *Significant vs BAS, ***ignificant vs BAS and ShA; $P<0.05$ for each comparison.

time-point and $\mathrm{F}_{2,127}=3.37 ; \quad P<0.05$ for the ANOVA including the 1400 time-point). As expected, a diurnal rhythm for CORT was observed with peak CORT levels at the 0700 time-point (ie just after the start of the dark/active phase) and lower levels measured approximately $7 \mathrm{~h}$ later at 1400. The ADX/C treatment regimen reproduced the circadian rhythm for CORT secretion but prevented increases in plasma CORT following either ShA SA, measured at 0930 immediately after the ShA SA session, or LgA SA, measured at 1400 immediately following the LgA SA session. Analyses of simple main effects at each timepoint in $\mathrm{ADX} / \mathrm{C}$ and sham-operated rats using one-way ANOVA followed by post hoc analyses showed that plasma CORT was significantly elevated following ShA or LgA SA in sham-treated, but not $\mathrm{ADX} / \mathrm{C}$ rats $(P<0.001)$. Elevations in CORT following ShA SA were transient and were restored to baseline levels within $5 \mathrm{~h}$ (ie before the 1400 blood sampling time).

Self-administration. Of the 18 rats that began the study in the ShA ADX/C, LgA pre-SA ADX/C, and LgA post-SA LgA $\mathrm{ADX} / \mathrm{C}$ groups, $13 \mathrm{ShA}$ ADX/C rats, $14 \mathrm{LgA}$ pre-SA ADX/C rats, and $15 \mathrm{LgA}$ post-SA LgA ADX/C rats completed SA testing. Figure 2 shows cocaine SA under basal conditions and across the 14 days of testing under ShA and $\operatorname{LgA}$ conditions in $\mathrm{ADX} / \mathrm{C}$ and sham-treated rats that underwent surgery before SA testing (ShA pre-sham, ShA pre-ADX/C, LgA pre-sham, and LgA pre-ADX/C rats) and in LgA rats that received $\mathrm{ADX} / \mathrm{C}$ after the 14-day SA period before extinction (LgA post-ADX/C rats). One-way ANOVA showed that rats in the various groups did not significantly differ in the number of days that they took to reach the training criterion for SA (ShA pre-sham $=8.19 \pm 0.83$; ShA pre-ADX/C $=8.54 \pm 0.93$; LgA pre-sham $=8.67 \pm 0.93$; LgA pre-ADX/C $=9.07 \pm 0.89$; LgA post- $\mathrm{ADX} / \mathrm{C}=8.60 \pm 1.10$ ) or in basal cocaine ShA cocaine SA before the 14 days of SA testing.

Separate two-way, ADX/C condition $\times$ SA day ANOVAs were performed for ShA and LgA rats. ADX/C before SA testing failed to significantly alter SA under ShA conditions. Two-way repeated measures ANOVA failed to show significant effects of SA day (1-14) or SA condition (ShA pre-SA sham vs ShA pre-SA ADX/C) on ShA SA or a significant SA day $\times$ SA condition interaction, suggesting that cocaine-induced CORT secretion is not necessary for

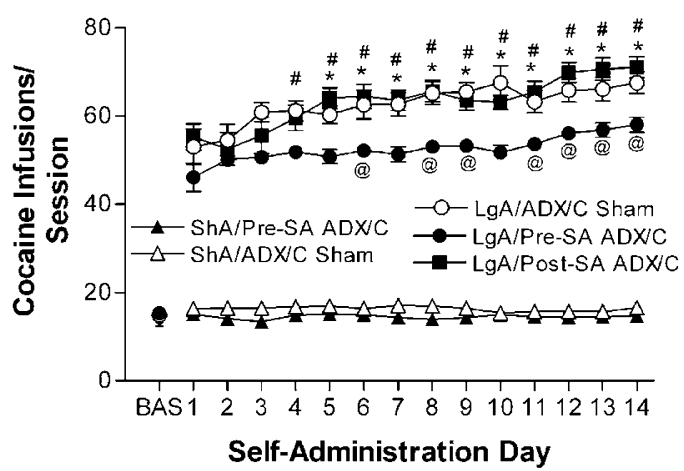

Figure 2 Effects of surgical adrenalectomy along with diurnal CORT replacement $(A D X / C)$ on cocaine SA under ShA and LgA conditions. Data represent the numbers of self-administered infusions under basal conditions and across the I4-day SA test period in ShA and LgA rats that underwent ADX/C before or upon completion of SA testing or received sham treatment. ADX/C before SA testing slowed, but did not prevent, the escalation of cocaine SA in LgA rats and had no effect on ShA SA (" significant vs LgA/Sham SA day I, *significant vs LgA/post-SA ADX/C SA day I, @ significant vs LgA/pre-SA ADX/C SA day I; $P<0.05$ for each comparison). Effects of ADX/C or time were not found in ShA rats.

ShA cocaine SA. By contrast, two-way repeated measures ANOVA revealed significant main effects of SA test day $\left(\mathrm{F}_{13,546}=19.65 ; P<0.001\right)$ and treatment group $(\mathrm{LgA}$ sham, LgA pre-SA ADX/C, LgA post-SA ADX/C; $F_{2,42}=7.87$; $P<0.001)$ on $\mathrm{SA}$ and a significant test day $\times$ treatment group interaction $\left(\mathrm{F}_{26,546}=1.57 ; P<0.05\right)$ in LgA rats. As expected, SA did not differ between LgA pre-SA sham and LgA post-SA ADX/C rats. However, overall, SA across the 14 days of testing was significantly reduced in rats that underwent $\mathrm{ADX} / \mathrm{C}$ before $\mathrm{SA}$ testing (LgA pre-SA ADX/C rats) compared to both LgA pre-SA sham and LgA post-SA $\mathrm{ADX} / \mathrm{C}$ rats $(P<0.05)$. Repeated measures ANOVA within each group showed that escalation of cocaine SA was found in all three LgA groups (LgA pre-SA sham, $F_{13,195}=6.89$; $P<0.001 ;$ LgA pre-SA ADX/C, $\mathrm{F}_{13,169}=6.77 ; P<0.001$; and LgA post-SA ADX/C rats, $\left.\mathrm{F}_{13,182}=9.35 ; P<0.001\right)$. Post hoc analyses showed that $\mathrm{SA}$ was significantly increased compared to day 1 of SA on days 4-14 in LgA pre-SA sham rats, days 6-9 and 11-14 in LgA pre-SA ADX/C rats, and days 5-14 in LgA post-SA ADX/C rats $(P<0.05$ for each comparison). Analysis of simple main effects on day one SA testing using one-way ANOVA showed that SA on day one of testing did not significantly differ among LgA pre-SA 
a
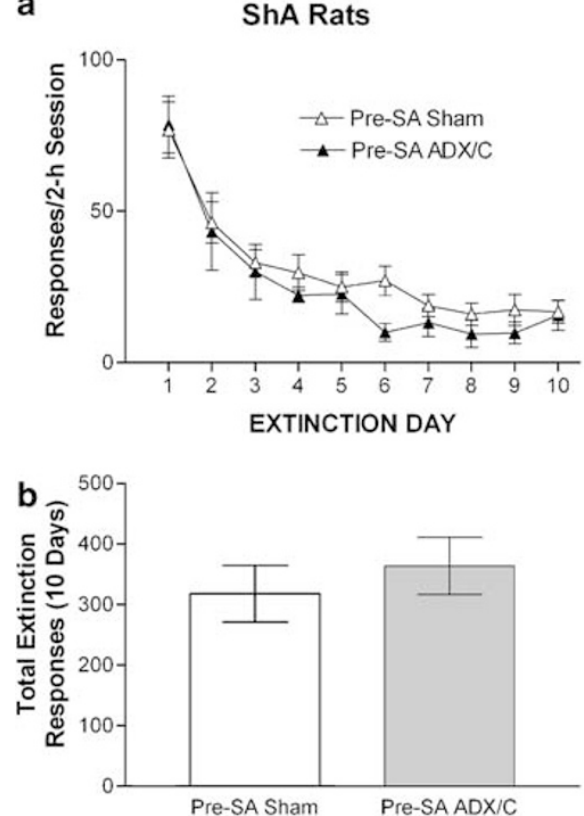

C
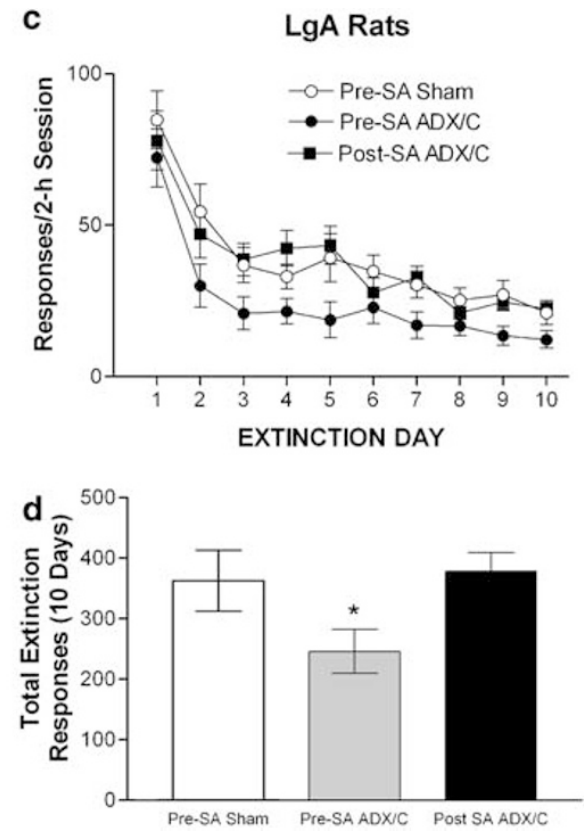

Figure 3 Extinction responding in sham-treated and ADX/C ShA (a and b) and $\operatorname{LgA}$ (c and d) rats. Data in (a) and (c) represent the daily mean numbers of responses on the previously active lever across the 10 days of extinction testing ( $\pm \mathrm{SE}$ ). Data in (b) and (d) represent the mean total numbers of responses (ie sum of responding across the 10 days) during extinction. ADX/C before, but not after, SA reduced extinction responding in LgA but not ShA rats. Total extinction responding was significantly reduced in LgA pre-SA ADX/C rats compared to both sham-treated LgA rats and LgA post-SA ADX/C rats (*significant $P<0.05$; (d)).

sham, LgA pre-SA ADX/C, and LgA post-SA ADX/C rats. By contrast, a significant effect of LgA SA group was observed on the final day of SA (ie day $14, \mathrm{~F}_{2,44}=8.70 ; P=0.001$ ). Altogether the data demonstrate that ADX/C before LgA SA attenuated, but did not prevent, escalating patterns of cocaine intake but had no significant effects on SA in ShA rats or SA on the first day LgA testing.

Extinction. Responding across the 10 days of extinction (3a and $3 \mathrm{c}$ ) and total extinction responding ( $3 \mathrm{~b}$ and $3 \mathrm{~d}$ ) in ShA and $\operatorname{LgA} \mathrm{ADX} / \mathrm{C}$ and sham-treated rats are shown in Figure 3. Extinction responding was unaltered in ShA rats that received $\mathrm{ADX} / \mathrm{C}$ before SA testing ( $3 \mathrm{a}$ and $3 \mathrm{~b}$ ). By contrast, ADX/C before, but not after, SA testing significantly reduced extinction responding compared to sham conditions in LgA rats ( $3 \mathrm{c}$ and $3 \mathrm{~d}$ ). A two-way LgA SA condition $\times$ extinction day (repeated measure) ANOVA showed significant main effects of extinction $\left(\mathrm{F}_{9,369}=42.94 ; P<0.001\right)$ and $\operatorname{LgA}$ SA condition $\left(\mathrm{F}_{2,41}=\right.$ $4.21 ; P<0.05)$ but no extinction $\times \operatorname{LgA}$ condition interaction. As expected, responding steadily declined across extinction testing in each of the $\operatorname{LgA}$ SA groups and was lowest on extinction day 10. Post hoc analysis demonstrated that LgA pre-SA ADX/C rats displayed significantly less extinction responding than both LgA pre-SA sham and LgA post-SA ADX/C ( $P<0.05$ for each comparison).

Reinstatement. Figure 4 shows reinstatement following an i.p. injection with cocaine $(10 \mathrm{mg} / \mathrm{kg})$ in ADX/C and shamtreated ShA and LgA rats. Cocaine-induced reinstatement was unaffected by ADX/C in ShA rats. Two-way repeated measures ANOVA in ShA ADX/C rats and sham controls showed a significant overall effect of reinstatement condi-

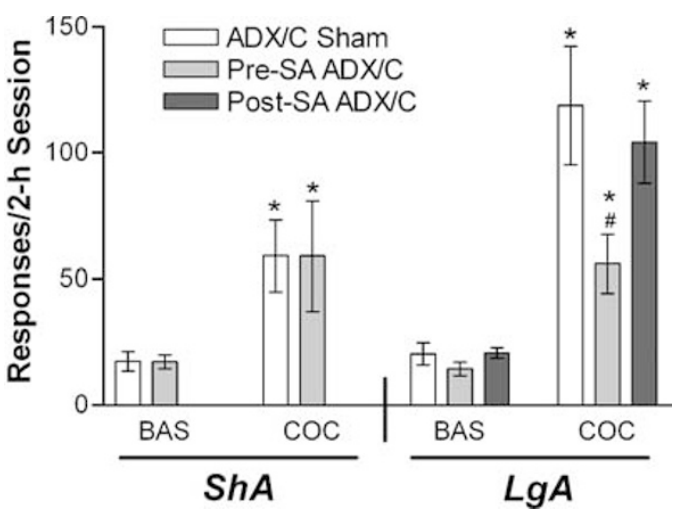

Figure 4 Cocaine-induced reinstatement in sham-treated and ADX/C $\operatorname{ShA}$ and $\operatorname{LgA}$ rats. Data represent mean cocaine lever responding $( \pm S E)$ during the reinstatement sessions following injection with cocaine (COC; $10 \mathrm{mg} / \mathrm{kg}$, i.p.) or saline (BAS) in sham- and ADX/C-treated ShA (left) and LgA (right) rats. Significant cocaine-induced reinstatement was observed in all treatment groups $(* P<0.05)$. ADX/C before $S A$ testing significantly attenuated cocaine-induced reinstatement in $\mathrm{LgA}$, but not ShA, rats (" significant vs ADX/C sham and post-SA ADX/C rat; $P<0.05$ ). By contrast, $A D X / C$ after $S A$ testing and before extinction failed to significantly alter cocaine-induced reinstatement in LgA rats.

tion $\left(\mathrm{F}_{1,28}=12.33 ; P<0.001\right)$, but no significant effect of $\mathrm{ADX} / \mathrm{C}$, and no significant reinstatement $\times \mathrm{ADX} / \mathrm{C}$ interaction. Significant reinstatement was observed in both ShA groups, but the magnitude of reinstatement did not differ between $\mathrm{ADX} / \mathrm{C}$ ShA rats and sham-treated controls, suggesting that (1) acute cocaine-induced increases in CORT are not necessary for cocaine-induced reinstatement, and (2) the neurobiological processes during SA by ShA rats 
that are responsible for establishing later reinstatement do not require elevated CORT.

By contrast, ADX/C before, but not after, the 14 days of SA testing, significantly reduced later cocaine-induced reinstatement in LgA rats. A two-way ANOVA showed significant main effects of reinstatement test condition (baseline $v s$ cocaine; $\mathrm{F}_{1,41}=66.29$ ) and $\mathrm{ADX} / \mathrm{C}$ condition (ADX/C sham, pre-SA ADX/C, and post-SA ADX/C; $\left.\mathrm{F}_{2,41}=3.14 ; P=0.05\right)$, and a significant interaction between reinstatement and $\mathrm{ADX} / \mathrm{C}$ conditions $\left(\mathrm{F}_{2,41}=3.29\right)$. Post hoc analysis showed that significant cocaine-induced reinstatement was observed in each of the $\operatorname{LgA}$ groups $(P<0.001$ for each). Separate one-way ANOVAs were performed to further examine differences in responding among the $\operatorname{LgA}$ groups under basal conditions and following cocaine. A significant effect of ADX/C condition was observed during the reinstatement session $\left(\mathrm{F}_{2,43}=3.27 ; P<0.05\right)$, but not under basal conditions (ie on extinction day 10). Post hoc testing showed that cocaine-induced reinstatement was significantly reduced in rats that received ADX/C before, but not after, SA testing compared to sham-treated controls $(P<0.05)$. Despite a statistically nonsignificant trend $(P=0.067)$, cocaine-induced reinstatement was not significantly reduced in rats that received $\mathrm{ADX} / \mathrm{C}$ before $\mathrm{SA}$ compared to rats that received $\mathrm{ADX} / \mathrm{C}$ after SA testing. Overall, these data suggest that elevated CORT at the time of SA testing is necessary for the augmentation of later cocaine-induced reinstatement that emerges in LgA rats.

\section{Experiment 3: Effects of Daily i.p. CORT Injections at the Time of ShA SA on Cocaine SA, Extinction, and Reinstatement}

Eight of the ten rats from the i.p. CORT treatment group completed SA testing. The effects of daily injection with i.p. CORT $(2.0 \mathrm{mg} / \mathrm{kg})$ before ShA SA across the 14 days of testing on cocaine intake and later extinction and cocaineinduced reinstatement are shown in Figure 5. As shown in Table 2, i.p. CORT elevated plasma CORT levels in a manner resembling LgA SA. Two-way repeated measures ANOVA failed to show significant effects of daily i.p. CORT pretreatment on cocaine SA under ShA conditions or on later extinction or cocaine-induced reinstatement, suggesting that although elevated CORT during LgA SA was necessary for the augmentation of later reinstatement and contributed to the escalation of cocaine intake, reproducing the increase in CORT observed during daily LgA SA through i.p. injections alone was insufficient to produce these responses in ShA rats.

Plasma CORT. The effects of a $2.0 \mathrm{mg} / \mathrm{kg}$ CORT injection before ShA SA on plasma CORT levels immediately after ShA SA or approximately $4 \mathrm{~h}$ later at a postinjection time corresponding to the end of a 6-h LgA session are shown in Table 2. Two-factor univariate ANOVAs were used to compare CORT levels in CORT pretreated ShA rats with CORT levels in untreated rats self-administering under ShA and LgA conditions. Since CORT samples were not acquired from LgA rats at the 0930 time-point, two separate ANOVAs were used: one examining CORT levels at 0700 and 0930 under basal conditions and in ShA rats with or without CORT pretreatment; and one examining CORT levels at
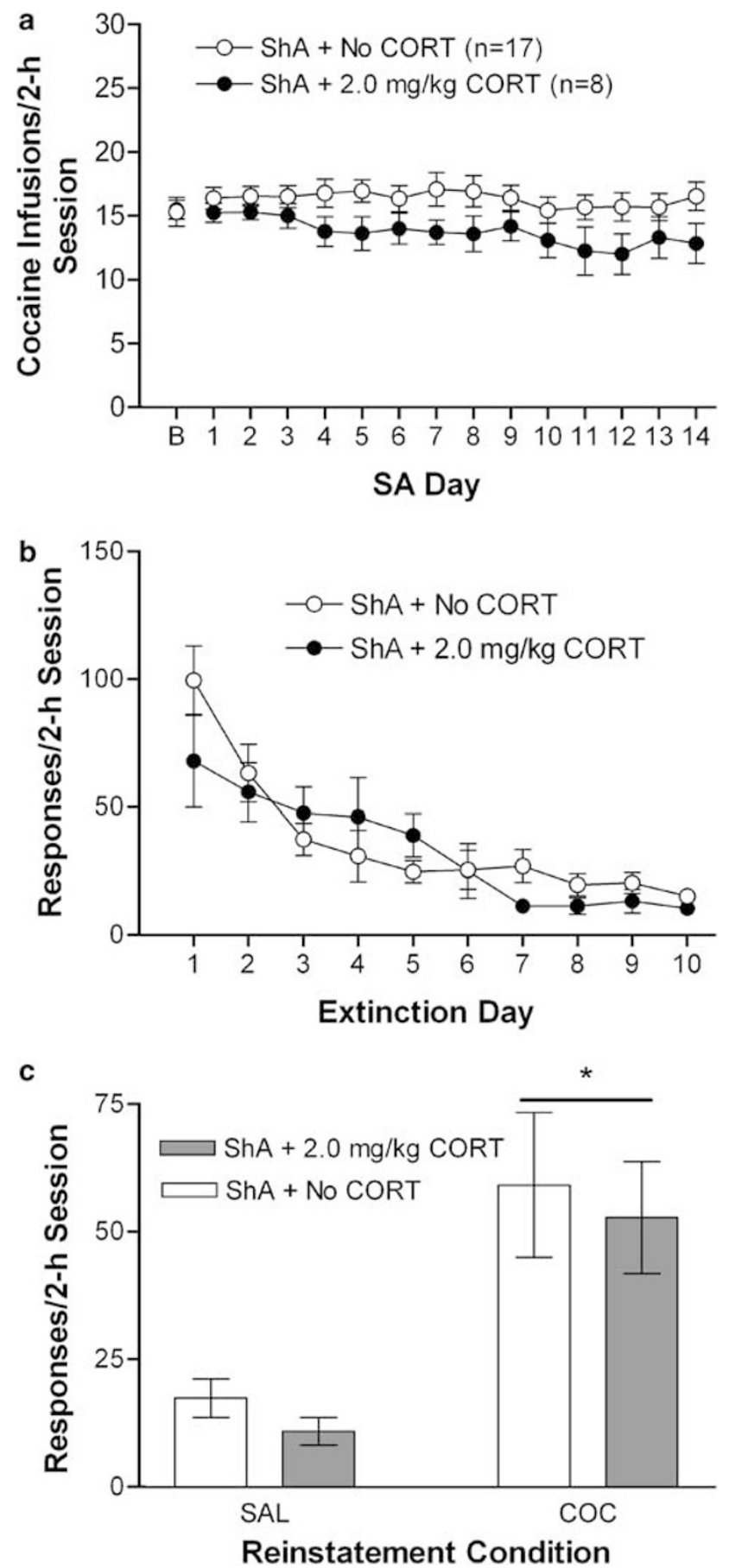

Figure 5 Cocaine SA (a), extinction (b), and cocaine-induced reinstatement (c) in CORT-pretreated rats tested for ShA SA for 14 days. The $2.0 \mathrm{mg} / \mathrm{kg}$ CORT dose was administered i.p. immediately before the daily ShA SA sessions. Data in (a) represent cocaine SA (mean infusions per session $\pm \mathrm{SE}$ ) under basal conditions (BAS) and across the 14 days of SA testing. Data in (b) represent daily responding across the I0-day extinction period. (c) Cocaine lever responding ( $10 \mathrm{mg} / \mathrm{kg}$, i.p.) during reinstatement testing is shown. Significant reinstatement was observed in rats tested earlier for SA with or without i.p. CORT injections $(* P<0.05)$. Differences in SA, extinction, and reinstatement were not observed between groups.

0700 and 1400 under basal conditions, in ShA rats with or without CORT pretreatment, and in LgA rats. In each case, a significant interaction between SA condition and time of 
testing was observed $\left(\mathrm{F}_{2,58}=9.84, P<0.001\right.$ for the 0930 ANOVA and $\mathrm{F}_{3,89}=4.68, P<0.01$ for the 1400 ANOVA). Post hoc analyses revealed that plasma CORT levels in ShA rats pretreated with i.p. CORT were significantly increased compared to basal conditions at 0930 and compared to basal conditions and to untreated ShA rats at 1400 $(P<0.05)$. No differences were found among groups at 0700. CORT levels in CORT-pretreated ShA rats did not significantly differ from untreated ShA rats at 0930 or from $\operatorname{LgA}$ rats at 1400 . Thus, by pretreating ShA rats with i.p. CORT, we were able to reproduce the pattern of CORT observed during LgA SA. Plasma CORT measured $12 \mathrm{~h}$ after the CORT injection (ie at 1900) was approximately $56 \mathrm{ng} / \mathrm{ml}$, down from $143 \mathrm{ng} / \mathrm{ml} 5 \mathrm{~h}$ early and close to basal levels determined in previous studies (eg Mantsch et al, 2003).

\section{DISCUSSION}

The primary finding of the present study is that surgical ADX along with diurnal CORT replacement before repeated SA testing slowed the escalation of cocaine SA and prevented the augmentation of cocaine-induced reinstatement resulting from SA under LgA conditions without affecting SA or reinstatement in ShA rats. We also report that CORT administration at a dose that mimics the plasma CORT response observed during LgA SA alone is insufficient to escalate SA or increase reinstatement in ShA rats. Our interpretation of these findings is that elevated glucocorticoids during cocaine use are necessary, but alone are insufficient, for drug-induced neuroplasticity thought to be critical for the onset of cocaine addiction. This permissive role for glucocorticoids in drug-induced neuroplasticity would be consistent with the findings that many physiological responses to stress depend on whether glucocorticoids are concurrently elevated (Sapolsky et al, 2000). Although apparently important for addiction-related neuroplasticity, we found that acute increases in glucocorticoids were not required for the acute reinforcing or reinstating effects of cocaine. We feel that these findings clarify the role of glucocorticoids in the addiction process and suggest that although stabilizing glucocorticoid levels may slow the process of becoming addicted, it is unlikely that eliminating glucocorticoid responsiveness will significantly impact cocaine-seeking behavior once addictionrelated neuroplasticity is already in place. Notably, this assertion is in line with clinical reports that the pharmacological inhibition of glucocorticoid secretion has little or no effect on cocaine use or cocaine-induced subjective effects in cocaine-dependent individuals (Ward et al, 1999; Kosten et al, 2002). Although we feel our observed effects were the consequence of a loss of a glucocorticoid response to cocaine SA during LgA testing, we cannot rule out possible effects stemming from the loss of other adrenal hormones following ADX.

\section{Escalation of SA and Augmentation of Cocaine-Induced Reinstatement in LgA Rats}

Consistent with previous findings (see eg Ahmed and Koob, 1998, 1999; Mantsch et al, 2004), a progressive escalation of cocaine intake was found in sham-treated $\operatorname{LgA}$, but not sham-treated ShA rats. It has been suggested that the escalation of cocaine intake in LgA rats may reflect the loss of control over drug use that is central to human cocaine addiction. As previously reported (Mantsch et al, 2004; Ahmed and Cador, 2006), reinstatement following a cocaine challenge was significantly augmented in sham-treated LgA rats compared to sham-treated ShA rats. By contrast, differences in responding on an inactive lever were not observed. Our findings add to a growing body of literature suggesting that the examination of cocaine SA under LgA conditions may provide insight into neuroplasticity that underlies the loss of control over cocaine use and chronically heightened susceptibility to drug relapse that are central features of human cocaine addiction. Despite the differences in SA and reinstatement, statistically significant differences in extinction responding were not observed between sham-operated ShA and LgA rats.

\section{Effects of LgA Cocaine SA on the HPA Axis}

We have previously reported that elevated CORT is necessary for the progressive escalation of cocaine SA that occurs with daily administration of a stressor, electric footshock, at the time of SA (Mantsch and Katz, 2007), suggesting that glucocorticoids play an important role in neuroplasticity that may be relevant to cocaine addiction. Cocaine SA, like stressors, increases circulating CORT levels in rats self-administering under both ShA and LgA conditions (Galici et al, 2000; Mantsch et al, 2003; Table 2). However, when rats self-administer cocaine under LgA conditions, increases in plasma CORT are more prolonged (Mantsch et al, 2003; Table 2), such that with repeated SA, LgA rats also show physiological signs of chronically elevated HPA activity, including enlarged adrenal glands and reduced thymus mass (unpublished observation). We hypothesized that the repetitively prolonged elevation of CORT resulting from daily LgA SA contributes to the neuroplasticity underlying escalating intake and heightened reinstatement that are observed in LgA rats. To investigate this, we examined cocaine SA, extinction, and reinstatement in rats that underwent surgical $\mathrm{ADX}$ along with diurnal CORT replacement (ADX/C; Mantsch and Katz, 2007). As expected, ADX/C selectively eliminated the CORT response to cocaine SA while maintaining normal diurnal patterns of CORT secretion.

\section{Effects of ADX/C on ShA and LgA Cocaine SA}

As we have previously reported, ADX/C failed to alter SA by ShA rats (Mantsch and Katz, 2007). The observed lack of effect of preventing glucocorticoid responses on cocaine SA is consistent with findings in nonhuman primates (Broadbear et al, 1999) and human cocaine addicts (Ward et al, 1999) that acute cocaine-induced increases in CORT secretion are not necessary for the acute reinforcing effects of cocaine. Others have demonstrated that complete elimination of glucocorticoids by surgical or pharmacological ADX in the absence of diurnal CORT replacement attenuates cocaine SA (Goeders and Guerin, 1996a,b; Deroche et al, 1997). However, as was the case in the present study, it has been reported that SA is restored when 
diurnal patterns of circulating CORT levels are reestablished (Deroche et al, 1997). A similar requirement for diurnal CORT secretion has been identified for the locomotorstimulating effects of cocaine (Marinelli et al, 1994).

ADX/C before SA testing also failed to significantly alter SA initially during $\operatorname{LgA}$ testing. However, the rate of escalation of cocaine intake in LgA pre-SA ADX/C rats was slower than in sham-treated controls, such that by day 14 of testing, cocaine intake was significantly reduced in the preSA ADX/C rats. Thus, it appears that although elevated glucocorticoids during LgA SA are not necessary for cocaine's acute reinforcing effects, elevated CORT appears to contribute to neuroplasticity that promotes the onset of escalating use patterns. Importantly, escalation was slowed, but not prevented, in LgA rats following ADX/C, suggesting that at least a component of the neurobiological response that underlies escalation is not glucocorticoid dependent.

\section{Effects of ADX/C on Cocaine-Induced Reinstatement in ShA and LgA Rats}

Similar to our findings with the escalation of cocaine SA, $\mathrm{ADX} / \mathrm{C}$ before SA testing significantly attenuated later cocaine-induced reinstatement only in $\operatorname{LgA}$ rats. In fact, LgA pre-SA ADX/C rats showed levels of reinstatement that were comparable to those in ShA rats, suggesting that elimination of the CORT response during LgA SA may have prevented the neuroplasticity responsible for increased susceptibility to relapse in LgA rats. By contrast, ADX/C after SA and before extinction failed to alter cocainereinstatement, indicating that elevated CORT during, but not after, the 14-day period of SA was necessary for the effects of LgA SA on reinstatement. Furthermore, the observations that $\mathrm{ADX} / \mathrm{C}$ after SA fails to alter reinstatement in LgA rats and that pre-SA ADX/C does not alter reinstatement in ShA rats indicate that acute cocaineinduced increases in circulating CORT levels are not necessary for cocaine-induced reinstatement. This assertion is consistent with reports in rats (Erb et al, 1998; Mantsch and Goeders, 1999), nonhuman primates (Lee et al, 2003), and human cocaine addicts (Ward et al, 1999) that cocaine-induced reinstatement or craving can be observed in the absence of acute elevations of circulating glucocorticoids.

Although one interpretation of the present findings is that ADX/C selectively prevents cocaine-induce neuroplasticity in $\operatorname{LgA}$ rats, an alternative interpretation is that the observed differences in cocaine-induced reinstatement may have been attributable to differences in earlier cocaine intake among groups rather than to altered patterns of glucocorticoid secretion. It has been reported that prior cocaine intake is a predictor of later reinstatement (Baker et al, 2001; Sutton et al, 2000). Since LgA pre-SA ADX rats self-administered less cocaine overall than LgA pre-sham rats, it stands to reason that any intake-dependent effects on reinstatement would be proportionally reduced. However, reinstatement did not differ between LgA pre-SA ADX rats and rats in the ShA SA groups despite a 2.5 -fold difference in cocaine intake. Thus, although it is possible that differences in cocaine intake contributed to the observed reductions in reinstatement, we consider it to be unlikely that drug intake during SA testing alone was responsible for the observed reinstatement effect.

\section{Extinction}

Overall, extinction responding was significantly reduced in $\mathrm{LgA}$ pre-SA ADX/C rats compared to sham-treated LgA rats and $\operatorname{LgA}$ rats that received $\mathrm{ADX} / \mathrm{C}$ after $\mathrm{SA}$ and before extinction. Pre-SA ADX/C failed to alter extinction responding in ShA rats. These findings are puzzling, considering that significant differences in extinction responding were not observed between sham-treated ShA and $\operatorname{LgA}$ rats, suggesting that $\mathrm{ADX} / \mathrm{C}$ was not simply preventing increases in extinction responding resulting from LgA SA. We have previously reported time-dependent alterations in extinction responding between ShA and LgA rats (Mantsch et al, 2004). It is unclear why such changes were not observed in the current study. Although it has been reported that glucocorticoids facilitate extinction learning (Yang et al, 2006), it is unlikely that the absence of elevated glucocorticoids during extinction contributed to the observed effect, since ADX/C effects on extinction were not observed in ShA rats and since $\mathrm{ADX} / \mathrm{C}$ after LgA SA but before extinction failed to affect extinction responding. Since we did see increased SA and reinstatement responding in LgA rats, we are reluctant to discount the possibility that although not observed in the present study increases in extinction responding do emerge following $\operatorname{LgA} \mathrm{SA}$ and are further reflective of cocaine-induced neuroplasticity, and that ADX/ $\mathrm{C}$ before SA testing also attenuated these effects. Further investigation is required to clarify this issue.

\section{Effects of i.p. CORT on ShA SA in ADX/C Rats}

Since eliminating the CORT response to SA in LgA rats slowed escalation and prevented the augmentation of later reinstatement, we hypothesized that cocaine-induced increases in CORT during SA were mediating the neuroplasticity that emerged in LgA rats. To test this hypothesis, we administered CORT to ShA rats before SA testing each day at a dose that produces an elevation of plasma CORT that is similar in magnitude and duration to that resulting from LgA SA. We have previously shown that a similar CORT administration regimen facilitates the acquisition of cocaine SA in rats (Mantsch et al, 1998). Similar to previous findings (Mantsch and Katz, 2007), CORT by itself failed to escalate cocaine SA in these rats and did not alter later cocaine-induced reinstatement, suggesting that although elevated CORT during LgA SA is necessary for addictionrelated neuroplasticity, comparable increases in CORT are incapable of escalating SA or augmenting later reinstatement in ShA rats. Interestingly, our earlier study demonstrating that $\mathrm{ADX} / \mathrm{C}$ prevented stressor-induced escalation of SA (Mantsch and Katz, 2007), we used electric footshock stress in ADX/C rats, implying that elevated CORT was necessary, but alone was insufficient for stressor-induced neuroplasticity and that glucocorticoids were facilitating the effects of one or more concurrently active neurobiological systems to produce escalation. Thus, we propose that elevated CORT during LgA SA plays a similar permissive role in the neuroplasticity underlying augmented cocaineseeking behavior in LgA rats. Identifying the neurobio- 
logical systems that work in concert with glucocorticoids to produce this neuroplasticity and those responsible for its expression should provide valuable insight into the neurobiological underpinnings of addiction as well as potential pharmacotherapeutic targets.

Notably, the lack of effect of repeated CORT administration on ShA cocaine SA in the present study differs from our previously published report that CORT administration under an identical regimen facilitated the acquisition of cocaine SA (Mantsch et al, 1998). There were a number of differences between the two studies that may have accounted for the disparate findings. Most importantly, whereas the present study examined the effects of CORT on cocaine SA after the acquisition of SA had already occurred, the earlier study examined the effects of CORT on SA by drug-naive rats during the acquisition phase.

Although the effects of ADX on cocaine-induced neuroplasticity are most likely attributable to the loss of adrenal glucocorticoids, we cannot rule out the possibility that elimination of other adrenal hormones, most notably aldosterone and dehydroepiandrosterone (DHEA) produced in the adrenal cortex and norepinephrine and epinephrine released from the adrenal medulla, contributed to our observed effects. CNS/behavioral effects of adrenal medullectomy have been reported (Borrell et al, 1983) and a role for norepinephrine in cocaine addiction has been proposed (Weinshenker and Schroeder, 2006). However, others have shown that selective removal of the adrenal medulla fails to alter norepinephrine levels within the central nervous system (CNS) (Peskind et al, 1986). DHEA administration has been reported to reduce cocaine SA and reinstatement (Doron et al, 2006), but the adrenal glands do not appear to be the source of DHEA in the brain (Corpechot et al, 1981). Although it is unlikely that a loss of aldosterone would directly contribute to our observed effects, we cannot rule out an indirect contribution of mineralocorticoid actions as a consequence of altered electrolyte balance, despite our attempts to stabilize sodium concentrations in our ADX rats through inclusion of $\mathrm{NaCl}$ in their drinking water. An understanding of the potential contributions of adrenal aldosterone, DHEA, and catecholamines, alone or in cooperation with glucocorticoids, to cocaine addiction-related neuroplasticity will require further investigation.

\section{Potential Targets for Glucocorticoid-Dependent Plasticity}

Glucocorticoid receptors (GRs) are widely distributed within the CNS (Ahima and Harlan, 1990) and regulate the activity of a broad range of neuronal systems (Joels and de Kloet, 1994), often via permissive effects (Sapolsky et al, 2000). These systems include those that mediate motivated behavior and serve as targets for abused drugs (Van Craenenbroeck et al, 2005). Although it is likely that elevated glucocorticoids promote adaptations within a number of neurobiological systems that contribute to cocaine-seeking behavior, the effects of persistently heightened glucocorticoids on the mesocorticolimbic dopamine (DA) system and its target cell populations may be particularly important to the addiction process. Glucocorticoids have been reported to regulate dopaminergic neuro- transmission (Piazza et al, 1996; Barrot et al, 2000), presumably through GR found within the cell bodies of dopaminergic neurons in the ventral tegmental area (VTA) (Harfstrand et al, 1986). GR activation has also been implicated in the strengthening of excitatory inputs into DA nerves in the VTA during times of stress (Saal et al, 2003). Notably, repeated binge pattern/LgA cocaine SA is associated with reductions in basal and stimulated DA release (Mateo et al, 2005; but see Ahmed et al, 2003), reduced presynaptic D2 autoreceptor sensitivity (Mateo et al, 2005), reduced inhibition of DA uptake by cocaine (Mateo et al, 2005; but see Ahmed et al, 2003), altered DA transporter binding (Ben-Shahar et al, 2006), and increased sensitivity to suppression of SA by the DA antagonist cis-flupenthixol (Ahmed and Koob, 2004). The dependence of these neuroadaptations on elevated glucocorticoids at the time of SA has not yet been investigated.

Also consistent with the apparent role of glucocorticoids as mediators of neuroadaptation (de Kloet et al, 2005; McEwen, 2005) and our findings that elevated CORT was involved in the induction, but not the expression, of addiction-related neuroplasticity are the structural changes within the mesocorticolimbic DA system that appears to emerge with chronic LgA cocaine SA. For example, it has been reported that LgA SA is associated with large increases in dendritic spine densities on neurons in the nucleus accumbens (Ferrario et al, 2005) and with changes in neuron-specific gap junction protein and mRNA expression in the nucleus accumbens, prefrontal cortex, and hippocampus (McCracken et al, 2005). The possibility that glucocorticoids are involved in these architectural changes in neuronal circuitry merits further investigation.

\section{CONCLUSIONS}

In summary, the present study defines a likely role for glucocorticoids in addiction-related neuroplasticity that emerges with repeated LgA cocaine SA. By contrast, shortterm cocaine-induced increases in glucocorticoids do not appear to be required for the acute reinforcing effects of cocaine or for cocaine-induced reinstatement. Although necessary for cocaine-induced neuroplasticity, elevated glucocorticoids alone appear to be insufficient to reproduce the effects of LgA SA on cocaine-seeking behavior, suggesting that glucocorticoids work in concert with other neurobiological systems that are active during cocaine SA, likely playing a permissive or enabling role. Understanding how glucocorticoids reshape the brain in a way that promotes neuroplasticity that is pathogenic for addiction and defining the neurobiological targets of this glucocorticoid-dependent neuroplasticity should provide valuable insight into the addiction process and aid in the development new approaches for the treatment of cocaine addicts.

\section{ACKNOWLEDGEMENTS}

This work was supported by National Institute on Drug Abuse (NIDA) grants number DA15758 to JRM and DA017328 to DAB. We thank Tanveer Sajan for her technical assistance and Dr Kristen Kau for her helpful comments on the manuscript. 


\section{DISCLOSURE/CONFLICT OF INTEREST}

Conflicts:

The authors declare that they have no conflict of interest related to this report.

\section{Professional affiliations:}

John R Mantsch and David A Baker are affiliated with Promentis Pharmaceuticals.

Joseph P Serge, Michael A Hoks, David M Francis, and Eric S Katz have no other professional affiliation at this time.

\section{REFERENCES}

Ahima RS, Harlan RE (1990). Charting of type II glucocorticoid receptor-like immunoreactivity in the rat central nervous system. Neuroscience 39: 579-604.

Ahmed SH, Cador M (2006). Dissociation of psychomotor sensitization from compulsive cocaine consumption. Neuropsychopharmacology 31: 563-571.

Ahmed SH, Koob GF (1999). Long-lasting increase in the set point for cocaine self-administration after escalation in rats. Psychopharmacology 146: 303-312.

Ahmed SH, Koob GF (1998). Transition from moderate to excessive drug intake: change in hedonic set point. Science 282: 298-300.

Ahmed SH, Koob GF (2004). Changes in response to a dopamine receptor antagonist in rats with escalating cocaine intake. Psychopharmacology 172: 450-454.

Ahmed SH, Lin D, Koob GF, Parsons LH (2003). Escalation of cocaine self-administration does not depend on altered cocaineinduced nucleus accumbens dopamine levels. J Neurochem 86: 102-113.

Baker DA, Tran-Nguyen LT, Fuchs RA, Neisewander JL (2001). Influence of individual differences and chronic fluoxetine treatment on cocaine-seeking behavior in rats. Psychopharmacology 155: 18-26.

Barrot M, Marinelli M, Abrous DN, Rouge-Pont F, Le Moal M, Piazza PV (2000). The dopaminergic hyper-responsiveness of the shell of the nucleus accumbens is hormone-dependent. Eur J Neurosci 12: 973-979.

Ben-Shahar O, Moscarello JM, Ettenberg A (2006). One hour, but not six hours, of daily access to self-administered cocaine results in elevated levels of the dopamine transporter. Brain Res 1095: 148-153.

Borrell J, de Kloet ER, Verteeg DH, Bohus B (1983). Inhibitory avoidance deficit following short-term adrenalectomy in the rat: the role of adrenal catecholamines. Behav Neural Biol 39: 241-258.

Broadbear JH, Winger G, Woods JH (1999). Cocaine-reinforced responding in rhesus monkeys: pharmacological attenuation of the hypothalamic-pituitary-adrenal response. J Pharmacol Exp Ther 290: 1347-1355.

Campbell UC, Carroll ME (2001). Effects of ketoconazole on the acquisition of intravenous cocaine self-administration under different feeding conditions in rats. Psychopharmacology 154: 311-318.

Corpechot C, Robel P, Axelson M, Sjovall J, Baulieu EE (1981). Characterization and measurement of dehydroepiandrosterone sulfate in rat brain. Proc Natl Acad Sci USA 78: 4704-4707.

de Kloet ER, Joels M, Holsboer F (2005). Stress and the brain: from adaptation to disease. Nat Rev Neurosci 6: 463-475.

Deroche V, Marinelli M, Le Moal M, Piazza PV (1997). Glucocorticoids and behavioral effects of psychostimulants. II. Cocaine intravenous self-administration and reinstatement depend on glucocorticoid levels. J Pharmacol Exp Ther 281: 1401-1407.

Doron R, Fridman L, Gispan-Herman I, Maayan R, Weizman A, Yadid G (2006). DHEA, a neurosteroid, decreases cocaine self-administration and reinstatement of cocaine-seeking behavior in rat. Neuropsychopharmacology 31: 2231-2236.

Erb S, Shaham Y, Stewart J (1998). The role of corticotrophinreleasing factor and corticosterone in stress- and cocaineinduced relapse to cocaine seeking in rats. J Neurosci 18: 5529-5536.

Ferrario CR, Gorny G, Crombag HS, Li Y, Kolb B, Robinson TE (2005). Neural and behavioral plasticity associated with the transition from controlled to escalated cocaine use. Biol Psychiatry 58: 751-759.

Galici R, Pechnick RN, Poland RE, France CP (2000). Comparison of noncontingent versus contingent cocaine administration on plasma corticosterone levels in rats. Eur J Pharmacol 387: 59-62.

Ghitza UE, Rothman RB, Gorelick DA, Henningfield JE, Baumann MH (2007). Serotonergic responsiveness in human cocaine users. Drug Alcohol Depend 86: 207-213.

Goeders NE, Guerin GF (1996a). Role of corticosterone in intravenous cocaine self-administration. Neuroendocrinology 64: $337-348$

Goeders NE, Guerin GF (1996b). Effects of surgical and pharmacological adrenalectomy on the initiation and maintenance of intravenous cocaine self-administration in rats. Brain Res 25: 145-152.

Harfstrand A, Fuxe K, Cintra A, Agnati LF, Zini I, Wikstrom AC et al (1986). Glucocorticoid receptor immunoreactivity in monoaminergic neurons of rat brain. Proc Natl Acad Sci USA 83: 9779-9783.

Jacobson L, Akana SF, Cascio CS, Shinsako J, Dallman MF (1988). Circadian variations in plasma corticosterone permit normal termination of the adrenocorticotropin responses to stress. Endocrinology 122: 1343-1348.

Joels M, de Kloet ER (1994). Mineralocorticoid and glucocorticoid receptors in the brain: implications for ion permeability and transmitter systems. Prog Neurobiol 43: 1-36.

Kippin TE, Fuchs RA, See RE (2006). Contributions of prolonged contingent and noncontingent cocaine exposure to enhanced reinstatement of cocaine seeking in rats. Psychopharmacology 187: 60-67.

Kosten TR, Oliveto A, Sevarino KA, Gonsai K, Feingold A (2002). Ketoconazole increases cocaine and opioid use in methadone maintained patients. Drug Alcohol Depend 66: 173-180.

Lee B, Tiefenbacher S, Platt DM, Spealman RD (2003). Role of the hypothalamic-pituitary-adrenal axis in reinstatement of cocaineseeking behavior in squirrel monkeys. Psychopharmacology 168: 177-183.

Mantsch JR, Goeders NE (1999). Ketoconazole does not block cocaine discrimination or the cocaine-induced reinstatement of cocaine-seeking behavior. Pharmacol Biochem Behav 64: 65-73.

Mantsch JR, Katz ES (2007). Elevation of glucocorticoids is necessary but not sufficient for the escalation of cocaine selfadministration by chronic electric footshock stress in rats. Neuropsychopharmacology 32: 367-376.

Mantsch JR, Saphier D, Goeders NE (1998). Corticosterone facilitates the acquisition of cocaine self-administration in rats: opposite effects of the type II receptor agonist dexamethasone. J Pharmacol Exp Ther 287: 72-80.

Mantsch JR, Yuferov V, Mathieu-Kia A-M, Ho A, Kreek MJ (2003). Neuroendocrine alterations in a high-dose, extended-access rat self-administration model of escalating cocaine use. Psychoneuroendocrinology 28: 836-862.

Mantsch JR, Yuferov V, Mathieu-Kia A-M, Ho A, Kreek MJ (2004). Effects of extended access to high versus low cocaine doses on self-administration, cocaine-induced reinstatement and brain mRNA levels in rats. Psychopharmacology 175: 26-36. 
Marinelli M, Piazza PV, Deroche V, Maccari S, Le Moal M, Simon H (1994). Corticosterone circadian secretion differentially facilitates dopamine-mediated psychomotor effect of cocaine and morphine. J Neurosci 14: 2724-2731.

Mateo Y, Lack CM, Morgan D, Roberts DC, Jones SR (2005). Reduced dopamine terminal function and insensitivity to cocaine following cocaine binge self-administration and deprivation. Neuropsychopharmacology 30: 1455-1463.

McCracken CB, Hamby SM, Patel KM, Morgan D, Vrana KE, Roberts DC (2005). Extended cocaine self-administration and deprivation produces region-specific and time-dependent changes in connexin36 expression in rat brain. Synapse 58: 141-150.

McEwen BS (2005). Glucocorticoids, depression, and mood disorders: structural remodeling in the brain. Metabolism 54: 20-23.

Meyer JS, Micco DJ, Stephenson BS, Krey LC, McEwen BS (1979). Subcutaneous implantation method for chronic glucocorticoid replacement therapy. Physiol Behav 22: 867-870.

Peskind ER, Raskind MA, Wilkinson CW, Flatness DE, Halter JB (1986). Peripheral sympathectomy and adrenal medullectomy do not alter cerebrospinal fluid norepinephrine. Brain Res 367: 258-264.

Piazza PV, Rouge-Pont F, Deroche V, Maccari S, Simon H, Le Moal M (1996). Glucocorticoids have state-dependent stimulant effects on the mesencephalic dopaminergic neurotransmission. Proc Natl Acad Sci USA 93: 8716-8720.

Prasad BM, Ulibarri C, Sorg BA (1998). Stress-induced crosssensitization to cocaine: effect of adrenalectomy and corticosterone after short- and long-term withdrawal. Psychopharmaco$\log y$ 136: 24-33.
Rouge-Pont F, Marinelli M, Le Moal M, Simon H, Piazza PV (1995). Stress-induced sensitization and glucocorticoids. II. Sensitization of the increase in extracellular dopamine induced by cocaine depends on stress-induced corticosterone secretion. J Neurosci 15: 7189-7195.

Saal D, Dong Y, Bonci A, Malenka RC (2003). Drugs of abuse and stress trigger a common synaptic adaptation in dopamine neurons. Neuron 37: 577-582.

Sapolsky RM, Romero M, Munck AU (2000). How do glucocorticoids influence stress responses? Integrating permissive, suppressive, stimulatory, and preparative actions. Endocrine Rev 21: 55-89.

Sutton MA, Karanian DA, Self DW (2000). Factors that determine a propensity for cocaine-seeking behavior during abstinence in rats. Neuropsychopharmacology 22: 626-641.

Van Craenenbroeck K, De Bosscher K, Vanden Berghe W, Vanhoenacker P, Haegeman G (2005). Role of glucocorticoids in dopamine-related neuropsychiatric disorders. Mol Cell Endocrinol 245: 10-22.

Ward AS, Collins ED, Haney M, Foltin RW, Fischman MW (1999). Blockade of cocaine-induced increases in adrenocorticotrophic hormone and cortisol does not attenuate the subjective effects of smoked cocaine in humans. Behav Pharmacol 10: 523-529.

Weinshenker D, Schroeder JP (2006). There and back again: a tale of norepinephrine and drug addiction. Neuropsychopharmacology [E-pub ahead of print; doi:10.1038/sj.npp.1301263].

Yang YL, Chao PK, Lu KT (2006). Systemic and intra-amygdala administration of glucocorticoid agonist and antagonist modulate extinction of conditioned fear. Neuropsychopharmacology 31: 912-924. 